\title{
Design and Implementation of Scenic Intelligent Guide System Based on WeChat applet
}

\author{
Jingwen $\mathrm{Li}^{1,2}, \mathrm{Xu} \mathrm{Li}^{2}$, Jianwu Jiang ${ }^{1,2, *}, \mathrm{Na} \mathrm{Yu}{ }^{2}$, Yuan $\mathrm{Ma}^{2}$, Wenda Chen ${ }^{2}$ \\ ${ }^{1}$ Guangxi Key Laboratory of Spatial Information and Geomatics, Guilin University of Technology, Guilin 541004, China \\ ${ }^{2}$ Guilin University of Technology, Guilin 541004, China
}

KEY WORDS: Intelligent tour guide system, WeChat applet, Mobile location,Route planning, Personalized push,Simulated annealing algorithm

\begin{abstract}
:
In view of the incomplete information acquisition, low visit efficiency and poor travel experience in the traditional tourism mode, under the background of the widespread use of WeChat, the intelligent guide system based on WeChat applet was designed and developed.Firstly, the overall architecture of the intelligent tour guide system is designed, including the basic data end, the network transmission end, the WeChat platform end and the application server.Secondly, the basic functions of the intelligent tour guide system are introduced.Finally, the key techniques for implementing the tour guide system are analyzed. The tour guide system is easy to use, simple to develop, has a good promotion and application prospect, and provides a new method and idea for the design of other travel service systems.
\end{abstract}

\section{INTRODUCTION}

Geographical awareness is a very important factor in smart tourism. Whether the geographical location information of tourists during the tourism process can be accurately and quickly obtained will directly affect the travel experience of tourists ${ }^{[1]}$.In the traditional tourism service, tourists obtain information about the surrounding environment through paper maps, scenic signs ${ }^{[2]}$, etc. This way of obtaining information is not only incomplete information acquisition, but also inefficient in visiting, far from meeting the diversified and high-level needs of tourists ${ }^{[3]}$. With the popularity of smartphones and the development of communication network technologies, mobile terminal devices have become an indispensable tool for tourists to travel. To this end, many domestic and foreign companies and research institutions have begun to develop travel apps based on mobile terminal devices.For example, the personalized travel app "Triposo"[4] developed by former Google employees can explore the historical scenery, artistic journey and natural scenery of the destination according to different routes, and can also push the attraction according to the preference information of the tourists."TripAdvisor"APP has become a worldrenowned travel data platform, with a large number of travel information from all over the world, sharing more than 500 million travel reviews and suggestions. With this app, travelers can not only get the most accurate and reliable travel information. You can also browse to the small, cost-effective tourist attractions.Domestic tourism APPs such as "Poor Travel" , "Ma Honeycomb Free Travel” and "Ctrip " cover a large number of tourist attractions, and all include hotel reservations, ticket inquiries and other services, which are favored by many tourists.

Although there are many kinds of travel apps, some apps do not have the smart service of pushing the surrounding attractions according to the current location of the tourists, and will occupy a large memory space of the mobile devices. In view of this, in order to meet the travel experience of tourists, in the context of the widespread use of WeChat, this paper developed a smart tour guide system based on WeChat small program, which provides convenience for tourists while providing personalized

* Jianwu Jiang - E-mail: fengbuxi@glut.edu.cn travel services for the tourism industry. The development is of great significance.

\section{WECHAT APPLET OVERVIEW}

WeChat applet is a new way for Tencent to connect users and services. It can be easily acquired and distributed in WeChat, and has excellent user experience ${ }^{[5]}$. The organization structure of small program page is shown in Figure 1.WeChat applets mainly use WXML (HTML5), WXSS (CSS) and JavaScript technology ${ }^{[6]}$. Users do not need to download or install to get services quickly, feature-rich, user experience is excellent, and provides a series of components and interfaces, rapid development, compared with traditional APP, WeChat applet has the following advantages:

(1) Simple development.WeChat applet provides a simple and efficient application development framework and rich components and APIs to help developers develop services with native APP experience on WeChat. The development process is relatively simple, programmers with front-end development experience. Can be seamlessly docked and quick start.

(2) User experience is convenient.WeChat applet does not need to be downloaded and installed, and can be directly opened by WeChat through sweeping, etc. After use, the applet will be automatically hidden, and the user experience is better.

(3) Technical maintenance and cost.The WeChat applet is based on the WeChat platform. Therefore, its maintenance is supported by WeChat official, with lower cost, shorter cycle and simpler process.

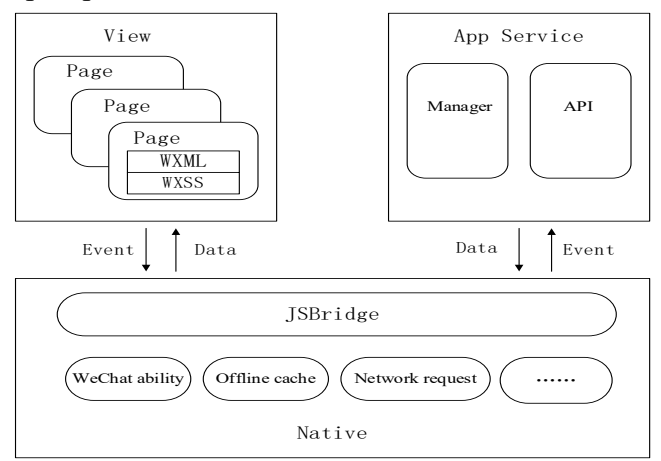

Figure1. WeChat applet structure diagram 


\section{OVERALL SYSTEM DESIGN}

\section{1 framework design}

The design goal of the intelligent tour guide system is to integrate various information such as geographic information, attraction introduction, tourism information and historical culture in the tourist scenic area. With the help of GIS, GPS and cloud push technologies ${ }^{[3]}$, a set of map navigation and attraction browsing based on WeChat platform is developed. Intelligent guide WeChat applet for information inquiry, service booking, online commenting and sharing ${ }^{[7]}$, providing visitors with graphical, visual and intelligent self-guided tour guide services. The whole system adopts a hierarchical design structure, which is divided into support layer, data layer, service layer and business layer from the bottom up, which is beneficial to the stability and scalability of the system. The system architecture is shown in Figure 2.
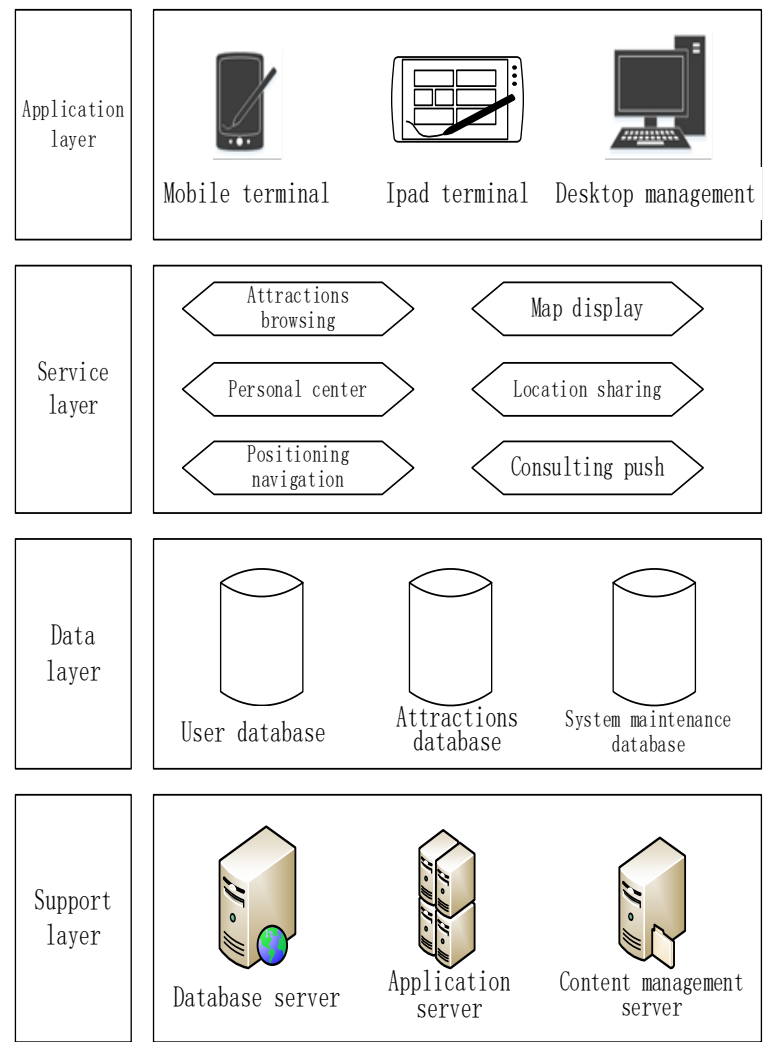

Figure 2. Intelligent tour guide system architecture

\subsection{Database Design}

The intelligent tour guide system uses Mysql database for data storage and management. The relationship between the database and the related objects of the attraction is shown in Figure 3 (in the figure, 1:N indicates a one-to-many relationship; M:N indicates a many-to-many relationship).

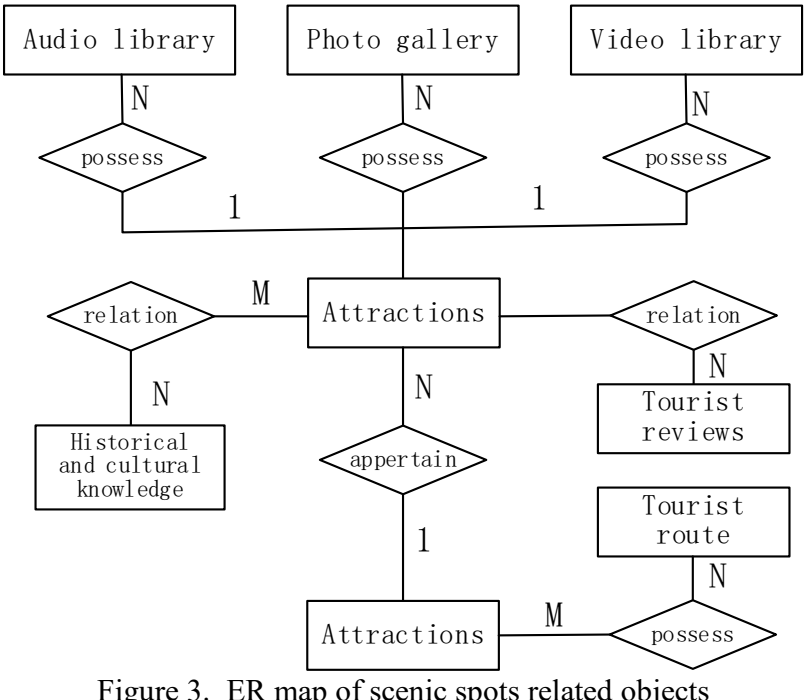

The Mysql database mainly includes two major categories: geospatial database and thematic attribute database. Geospatial databases primarily store geographic information such as attractions, roads, and surrounding buildings. The topic attribute database mainly includes attraction introduction, tourist information, surrounding transportation and dining accommodation. For the data with both spatial location information and thematic attributes, the coding structure of "color + ID" is designed to correlate in the geospatial database and the thematic attribute database. For example, the attraction is represented by green, the food is distinguished by orange, and the blue represents the mall. Each geographic information has other attributes, and its data table structure is shown in Table 1, 2 .

\begin{tabular}{|c|c|}
\hline Type number & types \\
\hline green & scenic spots \\
\hline Orange & fine food \\
\hline blue & emporium \\
\hline red & entertainment \\
\hline yellow & Hotel \\
\hline
\end{tabular}

Table 1. Attractions Type Table Structure

\begin{tabular}{|c|c|c|c|}
\hline Field & Description & Example & type of data \\
\hline ID & $\begin{array}{c}\text { Attractions } \\
\text { number }\end{array}$ & 000001 & $\operatorname{Int}(16)$ \\
\hline NAME & Spot name & Lijiang River & $\operatorname{Varchar(50)}$ \\
\hline TYPE & $\begin{array}{c}\text { Attractions } \\
\text { types }\end{array}$ & green & Varchar(50) \\
\hline LON & longitude & 110.438262 & $\begin{array}{c}\text { Decimal(10, } \\
6)\end{array}$ \\
\hline LAT & latitude & 25.153166 & $\begin{array}{c}\text { Decimal(10, } \\
6)\end{array}$ \\
\hline COMMENT & comment & 837 & $\operatorname{Int}(16)$ \\
\hline
\end{tabular}

Table 2. Tourist attraction data table structure 


\section{KEY TECHNICAL ANALYSIS AND FUNCTION DISPLAY}

\subsection{System implementation of key technologies}

4.1.1 Intelligent push of attractions based on A-GPS mobile positioning : Mobile positioning technology is the key to realize intelligent navigation functions such as visitor positioning, attraction push, and tour route analysis. At present, most smart guide apps make full use of the built-in GPS positioning module of the smartphone to realize the mobile positioning service ${ }^{[8]}$. However, since the general GPS positioning takes a long time to search for satellites that can be used in the current area, it is difficult to meet the demand for fast moving positioning ${ }^{[9]}$. To this end, an A-GPS (Assisted GPS) technology that combines network base station information and GPS information to quickly locate mobile terminals has emerged ${ }^{[10]}$. The A-GPS technology uses the network to provide GPS auxiliary information such as differential correction data and satellite operating status to the mobile terminal, thereby enhancing TTFF (TimeToFirstFix, the time required to send back the location information for the first time), so that the mobile terminal can quickly capture the GPS signal. And receiving the measurement information of the GPS satellite, and finally transmitting the measurement information to the positioning server in the network through the wireless network, and calculating the current location of the mobile terminal based on the received satellite navigation signal.

In order to implement A-GPS technology in attraction push, you first need to obtain permission. For example, for Android systems, first configure permission permissions in Androidfest.xml, for example: <uses-permission android:

Name $=$ "android.permission.ACCESS FINE LOCATION/>, <uses-permission android: name $=$ "android.permission. ACCESS COARSE LOCATION"/> where ACCESS_FINE_LOCA-TION indicates that GPS satellites can be used for precise positioning, ACCESS COARSE LOCATION indicates that it can be utilized The operator's mobile base station or Wi-Fi hotspot is used for coarse positioning. Then, the mobile positioning development interface provided by the mobile phone system is used for corresponding development.

4.1.2 Tourism Path Planning Based on Simulated Annealing Algorithm : The path planning problem is an indispensable part of the intelligent tour guide system. Its main function is to provide visitors with a solution to traverse the shortest trips of multiple attractions, which can reduce unnecessary travel expenses for tourists and save tourists. Part of the unnecessary time, so this function plays an important role in the entire tour guide system. Simulated Annealing Algorithm (SA), proposed by Metropolis et $\mathrm{a}^{[11]}$,, is an algorithm for solving optimization problems. It can perform fast optimization search, and use simulated annealing algorithm for tourism route customization, which can well solve tourism route planning. problem. The main steps are as follows:

(1) Set initial temperature $Q_{0}$, end temperature $Q_{e}$, Cooling rate $\mathrm{q}$ and number of iterations $\mathrm{X}$.

(2) Randomly generate a tourist route, including the tourist attractions to be visited, and calculate the fitness function value $\Phi\left(L_{0}\right)$.

(3) Perturb the current solution, generate a new solution, and calculate the new solution fitness function value $\Phi\left(L_{l}\right)$.
(4) Compare initial route fitness function value $\Phi\left(L_{0}\right)$ with new solution fitness function value $\Phi\left(L_{l}\right)$, if $\Phi\left(L_{0}\right)>\Phi\left(L_{l}\right)$, accept new travel itinerary $L_{1}$, Otherwise, accept new travel routes with a certain probability according to Metropolitic guidelines. (5) Determine whether the temperature drop termination condition is met. if $Q>Q_{e}$, Current temperature $Q=q \times Q$, and return to step (2), Otherwise output the optimal travel route.

\subsection{System function display}

The intelligent tour guide system has functional modules such as attraction recommendation, map display, and individual users. The system organization function results are shown in Figure 4.

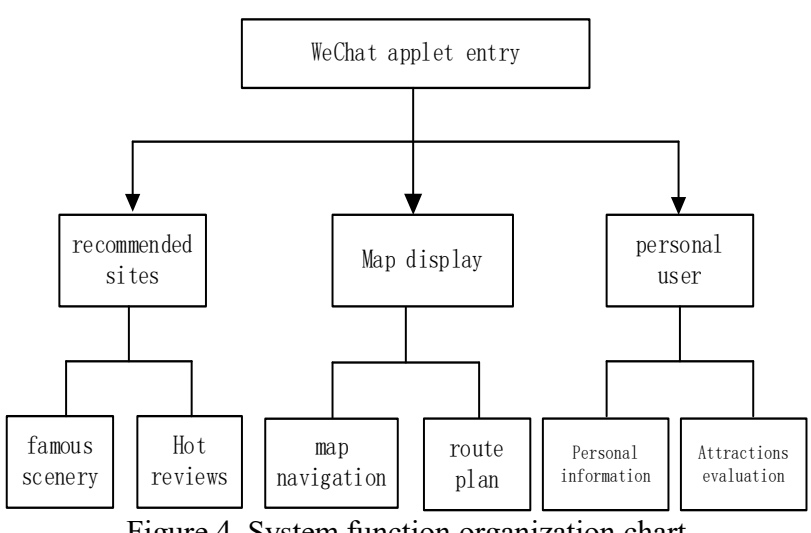

Figure 4. System function organization chart

4.2.1 Attractions recommendation function: The The attraction recommendation function is a new application based on location service. The system recommends the attraction by real-time positioning of the tourists. When the user arrives at a city or an attraction, the system will automatically recommend the local famous attractions and the surrounding attractions of the user. Visitors can also view other visitors' evaluations and introductions to the attraction, so that the user can make the user more convenient and detailed. Make a more optimized travel plan with information on the destinations of the destination. The scenic spot recommendation is shown in Figure 5 .

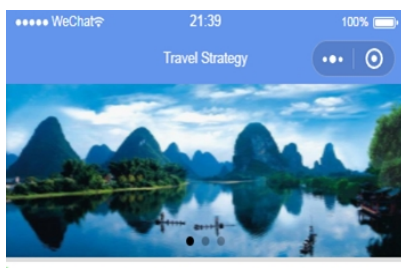

Attractions Recommended

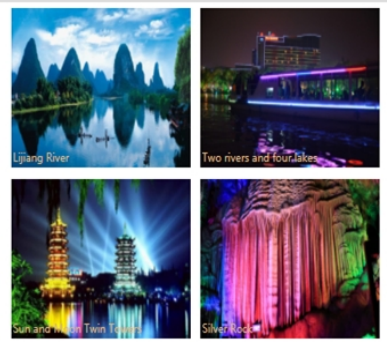

(a) Recommended hot spots

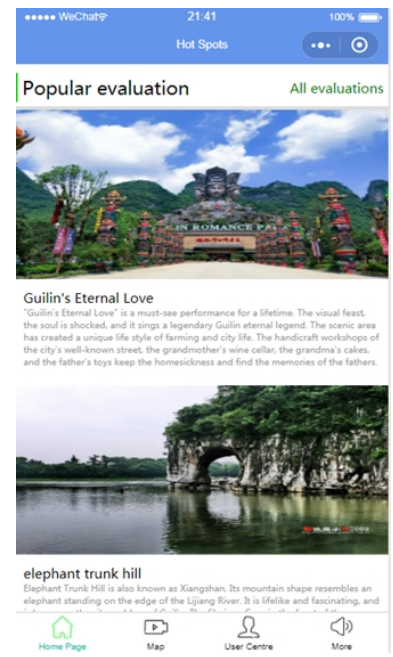

(b) Popular attractions
Figure 5. Sightseeing recommendation function diagram 
4.2.2 Tourism path planning : If someone wants to go to a city to play, ask for the shortest time, travel the least, and play as many places as possible, how should you plan your trip?In order to achieve this personalized travel requirement, we used a simulated annealing algorithm for travel route planning and introduced it into this tour guide system. We only need to select the destination you want to play in the MAP interface of the tour guide system, then input the expected time, expected consumption, and then click the route plan, the system will automatically generate a travel route for the tourists to meet the requirements of the tourists, as shown in Figure 6 . The recommendation of this personalized travel route plays a key role in improving the travel experience of tourists, making tourists more convenient.

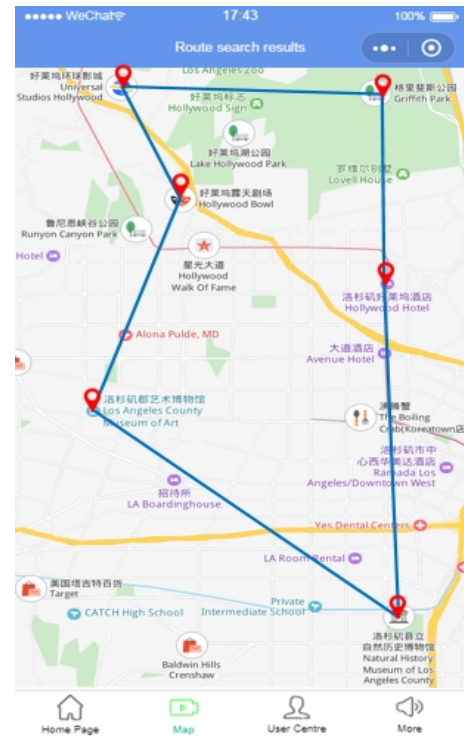

Figure 6. Tourism path planning

4.2.3 personal user: Users can register their personal accounts in the personal user interface or log in via WeChat or Alipay interface, which is convenient for viewing personal account management and viewing browsing trajectories.

\section{CONCLUSION}

The smart tour guide system based on WeChat applet designed in this paper makes full use of the characteristics of WeChat "open and ready, use and go, no download"[12], combined with A-GPS mobile positioning technology, personalized recommendation technology and travel path planning The technology realizes the various functions required for the smart scenic spot, so that it has a good promotion and application prospect. However, at present, the small program map interface lacks a good map mask function, and the mask cannot be scaled with the zoom of the map, which affects the design of the custom map to some extent. Although multi-party map providers provide good APIs, due to the encapsulation nature of small programs, it is impossible to maximize the cover function. I believe that in the future version update, you can see that the small program complements this defect, making it selfsufficient. Defining the map experience is even better.

\section{REFERENCES}

[1] Min Yang, Juntie Li,Liu Zhang.Tourism Information Science: A Research Framework[J]. Tourism Journal, 2011, 29(6): 72-79.

[2] Wei Liu,Meihui Xie,Wei Tian,Peichao Gao.The Tourism Application Model of WeChat Public Platform and Mobile Location Service[J].Science and Mapping Science, 2017, 42(01): 193-196+202.

[3] Wenyuan Zhang,Guoxin Tan,Chuanming Sun.Design and Implementation of a Scenic Intelligent Guide System[J]. Surveying and Mapping Geographic Information,2018, 43(05): 79-84.

[4] Triposo.Your Smart Travel Guide [EB/OL].[ 2016-07-08]. https://www.triposo.com

[5] Xueyun Zhang,Yan Yan,Jiubo Zhang.Design of Power Monitoring Management System Based on WeChat Small Program[J].Computer and Modernization,2017(12):98$102+107$.

[6] Tianle Kang, Wei Zhou, Zhen Qian, Jie Lu,Ziling Fan.The Service System of Qinyuan Intelligent Scenic Spot Based on WeChat Small Program[J].Journal of Nantong University(Natural Science Edition),2018,17(03):12-17.

[7] Nai Yang,Yang Li.Application of WeChat Small Program and Public Number in Field Geology Internship [J].Experimental Technology and Management,2019,36(02):246-251.

[8] Qunyong Yan,Aiguo Zhang,Qifeng Xu,et al. GPS mobile positioning and mobile network positioning accuracy analysis [J]. Global Positioning System, 2010, 35 (5): 33-37

[9] Zhangzhu Xu.Study on AGPS Localization Algorithm Based on Pseudo Code Phase/Pseudorange Combination[J].Journal of Gansu Sciences,2019,31(02):57-63.

[10] Leszczuk Agata,Chylińska Monika,Zi ba Emil,Skrzypek Tomasz,Szczuka Ewa,Zdunek Artur. Structural network of arabinogalactan proteins (AGPs) and pectins in apple fruit during ripening and senescence processes.[J]. Plant science : an international journal of experimental plant biology,2018,275.

[11] Dantzig G B,Ramser J H.The Truck Dispatching Problem[J].Management Science,1959,6(1):80-91

[12] Heping Shi,Yaping Liu.Development of Tourist Service Robots Based on WeChat Platform[J].Journal of Xuzhou Institute of Technology(Natural Science Edition), 2018,33(01): 88-92. 